\title{
Visual Reminders and Patient Compliance of Type 2 Diabetics
}

\section{Thomas A Clobes}

Faculty of Healthcare Administration, Colorado State University Global Campus, 7800 East Orchid St., Suite 200 Greenwood Village CO 80111 , USA

Corresponding author: Thomas A Clobes, Faculty of Healthcare Administration, Colorado State University Global Campus, 7800 East Orchid St., Suite 200 Greenwood Village CO 80111, USA, Tel: 805-665-3765; E-mail: thomas.clobes@csuglobal.edu

Received date: March 29, 2017; Accepted date: April 11, 2017; Published date: April 18, 2017

Copyright: @ 2017 Clobes TA. This is an open-access article distributed under the terms of the Creative Commons Attribution License, which permits unrestricted use, distribution, and reproduction in any medium, provided the original author and source are credited.

\begin{abstract}
Objective: To determine how visual reminders affect patient compliance in Type 2 diabetics following the completion of a disease management program, a pretest-posttest randomized design was used.

Methods: Patients were recruited following enrollment in a diabetes management course. Classes were randomized into the visual reminder group (VG) or control group (CG). The VG selected an object of importance and one seen frequently, reminding themselves to choose healthy behaviors. Baseline and follow-up data included $\mathrm{HbA1c}, \mathrm{BMI}$, and exercise time.

Results: For $\mathrm{HbA1c}$, the decrease in the CG was $5.49 \%(0.56 \pm 1.4)$ while the VG group was $13.3 \%(1.2 \pm 1.6)$ with no statistical significance between the two groups $(p=0.36)$. From baseline to follow-up, the change in the $C G$ was not statistically significant $(p=0.3)$ while the change in the VG was $(p=0.04)$. For BMI, the CG reduced $0.53 \%$ while the mean reduction with the VG group was $1.5 \%$. The difference between the groups was not statistically significant $(p=0.61)$. For exercise time, the CG was increased $18.4 \%$ while the mean increase in the VG group was $82.0 \%$. The difference was not statistically significant $(p=0.27)$. The change from baseline for both the CG and VG $(p=0.004$ and 0.02 , respectively).

Conclusion: Though trending positively, this research cannot conclusively confirm the value of a visual reminder in changing patients' health behavior to manage their diabetes. Nonetheless, when considering the clinically significant differences between the CG and VG with greater improvements in all measured variables, implementation of a visual reminder into diabetes management should be considered.
\end{abstract}

Keywords: Diabetes; Type 2 diabetes; Patient compliance; Disease management; Patient outcomes

\section{Introduction}

Type 2 Diabetes Mellitus (T2DM) is a challenge for patients, physicians, and healthcare organizations. Risks patients and physicians deal with include serious complications of nephropathy, retinopathy, and amputation [1]. To successfully manage the disease, patients must control their diet, prick their fingers throughout the day, exercise, and take their medication [2]. From a managed care perspective, $12.3 \%$ of adults older than 20 years of age and $25.9 \%$ of those over the age of 65 have diabetes, a considerable increase over the past decades [3-5]. Likewise, $14 \%$ of healthcare spending goes to treating it, amounting to hundreds of billions of dollars a year, and nearly $95 \%$ of diabetes cases are type 2 [6,7]. Lowering glycated hemoglobin (HbAlc) through disease management programs improve patient outcomes and with clinically significant benefits from even small reductions [8-15].

Disease management programs attempt to treat a disease comprehensively. With diabetes, disease management includes patient education, clinician guidelines, regular laboratory tests, referrals to podiatrists and ophthalmologists as needed, provision of glucosetesting supplies, and so forth [16]. Providers benefit from disease management programs because of the assistance from other members of the healthcare delivery system and improved patient outcomes [13] Managed care organizations benefit not only from the improved patient outcomes, but also from reduced patient costs. Providers may be ordering more laboratory testing and patients may be seeing more specialists, but cost savings result from reducing the long-term complications.

Current research on disease management has been focusing on novel techniques of administering programs such as delivery methods and provider training. The overall success of disease management programs has led researchers to inquire if better results can be reached with various administrations. However, little research has focused on methods for daily reinforcement of the material learned in disease management programs. The data have demonstrated that disease management programs can be effective, but there is still room for improvement; patients, providers, and health plans alike need to identify other tools to further improve patient outcomes [9-15].

There is limited research on the use of visual reminders to change health behavior. However, this technique has been evaluated in areas such as sunscreen use, smoking cessation, and dieting [17-19]. The literature is lacking in terms of evaluating this technique in the application of a disease management program or with T2DM as the focus. The goal of this study was to determine if the use of a visual reminder can improve patient compliance in Type 2 diabetics following the completion of a disease management program. 


\section{Methods}

\section{Study design and analysis}

The Wellness Center (TWC) is an out-patient clinic of San Joaquin Community Hospital in Bakersfield, CA, with patients referred from community physicians. The diabetes educational series is one routinely administered at this site by Certified Diabetes Educators, Registered Nurses, and Registered Dieticians. This consists of four, two-hour courses on an introduction to diabetes, medications, meal planning, and exercise.

Subjects were selected from those who had enrolled in the series at TWC. Classes as a whole, not as individual subjects, were randomly assigned to the control group (CG) or visual reminder group (VG) to test the hypothesis that a visual reminder would encourage behavior changes following completion of the series as measured by $\mathrm{HbAlc}$, body mass index (BMI), and exercise time. Inclusion criteria consisted of enrollment and completion of the educational series, a diagnosis of T2DM, and age of 18 or older. Exclusion criteria included a baseline $\mathrm{HbA1c}$ level of less than $6.5 \%$ or a diagnosis of any form of diabetes other than T2DM. Pregnant women were excluded.

The expected sample size necessary, using the independent samples t-test, to conduct a valid statistical analysis was 14 , seven in each group. This calculation was done using the statistical software $\mathrm{G}^{*}$ Power [20]. This was confirmed with using a standard t-test sample size formula [21]. A two-tailed analysis was selected. An alpha of 0.05 and beta of 0.2 were used to make this study as robust as possible from a statistical perspective. An effect size of 1.67 was calculated for a change in $\mathrm{HbA1c}$ level in the intervention group of 0.5 higher than the CG with a standard deviation of 0.3 . A reduction in $\mathrm{HbAlc}$ of 0.5 would be clinically meaningful [8]. The choice of 0.3 for the standard deviation of the mean difference was a logical guess, derived from the wide range of initial and follow-up values in the literature on diabetes management [9-15]. TWC experiences an average drop-out rate of patients who do not complete the entire four courses of $10-20 \%$ (K. Wiggins, personal communication, June 14,2010$)$. It was expected to lose a comparable amount after that course series was completed who would not return for their follow-up visit. Therefore, a minimum of ten subjects per group, for a total of 20 , were recruited.

Baseline data, measured during the pretest, included HbAlc, BMI, and minutes of exercise per week. After randomization, on the first day of the class series, the VG group was read the motivational statement:

You will learn in this class that controlling your diabetes with regular exercise, a proper diet, and use of physician-prescribed medication is very important. How well you control your diabetes will have a direct impact on long-term complications you experience because of it.

This research is designed to identify ways to help you control your diabetes. In order to do this, you need to select a visual reminder you see on a daily basis. This should be something that evokes positive emotions and feelings for you; you want this visual reminder to be a source of encouragement to you. Next week, you will be asked to share this visual reminder and affirm the statement: "When I look at (my visual reminder), I will remember to make good behavior choices that will help me control my diabetes."

The VG was also asked to select an object they see daily that was of importance to them to serve as a reminder of what they learned in the course. The following class session, each subject identified their selected visual reminder and made a commitment to changing health related behaviors that would improve their diabetes management. Subjects then continued on with the course as usual. Follow-up data was collected 3 months following completion of the educational series; the VG was also asked at the follow-up the frequency they saw their visual reminder (Frequently: three or more times each day; Often: three or more times, most days; Sometimes: one or two times, most day; Rarely: less than once per day; or Never) and perceived influence it had on altering their behaviors (Significant: it reminded me to make healthy choices and I did so all or most of the time; Great: it reminded me to make healthy choices, and I did so most of the time; Some: it reminded me to make healthy choices and I did so some of the time; Little: it reminded me to make healthy choices, but I did not do so; or None: it did not remind me to make healthy choices).

Statistical significance between the two groups of the HbAlc, BMI, and exercise time data was analyzed with an independent samples ttest. To determine a significant difference from baseline to follow-up within each group, a paired sample t-test was performed.

\section{Instrumentation validity and reliability}

Measurement of HbAlc levels was done with the DCA 2000+ Analyzer from Bayer which has shown to have comparable results to standard laboratory methods [22].

\section{Ethics review}

The methods and ethics of this study were evaluated by the Institutional Review Board at The Rutgers School of Health Related Professions.

\section{Results}

\section{Subject enrollment}

The response rate from TWC patients willing to participate was generally high at $44 \%$. The majority of those who declined participation were male, $68 \%$ of those asked, or African-American, $71 \%$ of those asked. During the enrollment period, 27 subjects volunteered and had their $\mathrm{HbAlc}$ analyzed. Four subjects were excluded because their $\mathrm{HbAlc}$ was too low to meet the study inclusion criteria. One subject's analysis was unsuccessful and refused a second finger prick. Twenty-two subjects continued with the study and began the education series at TWC. Three subjects did not complete the course series and, despite given the opportunity, never returned to complete the courses missed. Two subjects did not return for their follow-up analysis. Final subject count was 17: 9 in the VG and 8 in the CG.

\section{Baseline characteristics}

Table 1 shows the baseline characteristics of the two groups, which did not vary significantly. The most noted differences were with sex and diagnosis time. The VG contained markedly more men and fewer women than the CG. Similarly, the CG had a noticeably longer time since their initial diagnosis than the VG. The CG and VG were both primarily made up of Caucasian subjects $(75 \%$ and $66.7 \%$, respectively) but also included Hispanic (25\% and $22.2 \%)$ as well as African American (0\% and 11.1\%). 


\begin{tabular}{|l|l|l|l|}
\hline Characteristic & \multicolumn{2}{|l}{ Group } & P Value \\
\hline & CG & VG & \\
\hline Mean Age & $63 \pm 11.5$ & $54.3 \pm 10.5$ & 0.14 \\
\hline Male\% & 37.5 & 55.6 & \\
\hline Mean Diagnosis Time (years) & $8.3 \pm 9.0$ & $3.9 \pm 5.9$ & 0.25 \\
\hline Mean HbA1c & $7.8 \pm 1.4$ & $8.3 \pm 1.5$ & 0.49 \\
\hline Mean BMI & $34.9 \pm 5.3$ & $36.4 \pm 3.9$ & 0.51 \\
\hline Mean Exercise Time (mins/week) & $88.1 \pm 73.3$ & $61.7 \pm 57.3$ & 0.51 \\
\hline
\end{tabular}

Table 1: Subjects baseline characteristics.

\section{Visual reminders}

A narrow range of visual reminders was selected, mostly either photographs of family members or actual individuals of meaning to the subjects. Further, reasons for selecting a given reminder were generally from a motivational aspect for being healthy for loved ones. A frequency of seeing their visual reminder of "Sometimes," "Often," or "Frequently" was reported by $77.8 \%$ of the subjects, with $44.4 \%$ reporting "Often" or "Frequently." An impact on one's changing one's behavior of "Some," "Great," or "Significant" was reported by $77.8 \%$ of the subjects, with $55.6 \%$ reporting "Great" or "Significant."

\section{Blood glucose}

Both groups had a reduction in HbAlc. The mean reduction with the CG was $5.49 \%(0.56 \pm 1.4)$ while the VG group was $13.3 \%(1.2 \pm$ 1.6; Figure 1). There was no statistical significance between the two groups $(\mathrm{p}=0.36)$. A reduction was observed in $62.5 \%$ of the subjects in the CG and $77.8 \%$ in the VG group. Of those subjects, $50 \%$ and $55.6 \%$, respectively, had a clinically significant reduction [8]. Further, $12.5 \%$ and $55.6 \%$ lowered their HbA1c enough to meet the goal established by the American Diabetes Association (ADA) of 7.0\% [23].

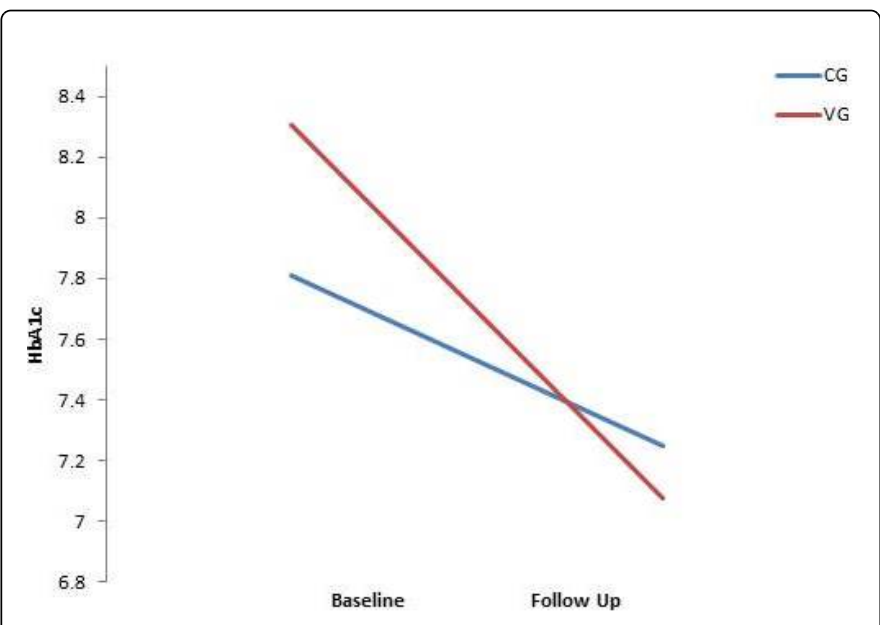

Figure 1: Change in HbAlc for CG and VG.

Though the results were not statistically significant between the two groups, a paired sample t-test revealed a non-statistically significant change in the CG $(p=0.3)$ while the change in the VG was

statistically significant $(\mathrm{p}=0.04)$. However, the change for all subjects as a whole was $9.6 \%(0.92 \pm 1.5)$ and was a statistically significant change $(\mathrm{p}=0.02)$.

\section{BMI}

Both groups experienced a reduction in BMI. The CG reduced their BMI by a mean of $0.53 \%(0.19)$ while the mean reduction with the VG group was $1.5 \%(0.54)$. The difference between the groups was not statistically significant $(\mathrm{p}=0.61)$. A paired sample $\mathrm{t}$-test revealed neither group had a reduction that was statistically significant at follow-up versus baseline ( $\mathrm{p}=0.78$ and 0.28 , respectively).

\section{Exercise time}

There was an increase in weekly exercise time in both groups. The mean increase with the CG was $18.4 \%$ ( 6.3 minutes per week) while the mean increase in the VG group was $82.0 \%$, (50.6 minutes per week). The difference was not statistically significant $(\mathrm{p}=0.27)$. The paired samples t-test revealed a statistically significant change from baseline for both the CG and VG ( $\mathrm{p}=0.004$ and 0.02 , respectively). Likewise, there was a statistically significant change for all subjects as a whole $(\mathrm{p}=0.04)$.

\section{Discussion}

\section{HbA1c}

The hypothesis was not conclusively supported by the data gathered in this study. These results conflict with data from other studies using a visual reminder. Previous research has shown that both positively and negatively phrased visual messages can affect health related behaviors and attitudes, specifically related to smoking [19]. In their work, subjects' attitudes toward smoking as well as cigarette consumption improved with either phrased message, but more so with positively worded messages. This is similar, compared to the current research, to the motivational statement subjects read and affirmed. Though the effects on a printed visual reminder had impacts on nonsmoker's and smoker's attitudes, the same was not seen with T2DM patients.

Some of the inconsistencies could be explained by the notable difference in the two groups of percentage of male participants. The CG was made up of $37.5 \%$ male subjects while the VG group had $55.6 \%$ male. Men are less likely to wash their hands following restroom use even with a visual reminder to do so [24]. In fact, in this specific example, men's hand washing behavior did not differ in the presence or absence of a visual reminder. Since the VG group had a markedly higher percentage of men than the CG, it is possible this affected the results.

Another explanation for the difference could be the type of visual reminder, frequency of exposure to the reminder, and length of time between initial exposure and measurement of behavior change. In the other studies, the visual reminder was generally some variation of a message reinforcing the behavior change goal $[17,19,24]$. Conversely, this research asked subjects to pick a meaningful item to them as a positive reminder and had frequent exposure, possibly reducing the effectiveness of the message behind the reminder. Additionally, all of the studies, with the exception of Schneider et al. [19], did not determine a behavior change beyond the immediate period of time after initial exposure. However, other research has examined the efficacy of frequently viewed reminders. Fox [25] reported the impact 
on fruit and vegetable sales at grocery stores near billboards with pictures of a fruit or vegetable and the health message "eat five a day for better health." Stores nearest the billboard had an increase in sales of $4 \%$ to $20 \%$ versus the same time period the previous year. Though there was no measurement on the frequency of which consumers viewed the billboard, the visual reminder in Fox et al. report is closer to what was evaluated in this work in regards to frequency, suggesting that the impact of the message does not necessarily deteriorate over time. Nonetheless, the results were not consistent.

Both groups had clinically significant reductions in $\mathrm{HbAlc}$, greater than 0.5 , and the VG group's reduction was more than double what was observed from the CG [8]. Additionally, more subjects in the VG group had a clinically significant reduction and far more met the ADA goal [23]. Subjects also reported a fairly high amount of impact from viewing their visual reminder. The hypothesis was supported to a degree by the marked difference between the two groups, despite not reaching statistical significance.

The lack of a statistical significance and inconsistencies with previous research can largely be explained by the small sample size and the variability of $\mathrm{HbAlc}$ between subjects. Though the sample size was larger than needed and the difference between the two groups was greater than expected, the variability was much greater than anticipated. Repeating the sample size calculation, again using the statistical software $G^{\star}$ Power, keeping all variables the same as used prior to commencing the research, but replacing the effect size of 1.67 with 0.46 based upon the actual variability observed, the required sample size increases over ten-fold to 152 [20].

\section{Secondary variables}

Both the CG and VG had a reduction in BMI throughout the duration of the study, though the VG's percentage of BMI decrease was nearly three-fold the CG's. Though the difference between the two was not statistically significant and the change from baseline to follow-up was also not, the clinical significance of a nearly three-fold difference in BMI change supports the role of a visual reminder in patients changing behaviors that lead to weight loss. Any issues with the sample size aside, there was not enough of a time span from the baseline to follow-up measurements. An observational study, grouping subjects based on their intention to lose weight or not showed an increase in BMI over one year [26]. Statistically significant reductions in BMI have been observed in a period of just two months, but when the intervention being studied specifically focused on weight loss [27]. The data indicate a trend supporting the use of a visual reminder that may have been statistically significant with a longer period of time.

Given the large changes in minutes per week of exercise (18.4\% for the CG \& $82.0 \%$ for the VG) and the lack of statistical significance between the two, this variable perhaps suffered most from the small sample size. This is further supported by the statistical significance within each group and the totality of subjects from baseline to followup. Likewise, the markedly higher increase with the VG is consistent with prior research changing health related behaviors with a visual reminder [17,19].

\section{Limitations and further research}

The most significant weakness of this study is the small sample size. Due to the small sample and the high variability that was observed, it is not possible to reach a conclusive decision on the value of a visual reminder in improving T2DM patients' compliance. However, this is easily resolved through additional research with larger subject samples.
Less significant weaknesses include the omission of other important variables to diabetes management: blood pressure, medication adherence, regular check-ups with various specialists, and the like. This was beyond the scope of this study and was not included because the $\mathrm{HbA1c}$ measurement was considered representative of overall patient compliance and reflective of a subject's risk level [8]. Additional research could be carried out evaluating the impact of a visual reminder on these specific variables. Conversely, strength of this study was evaluating a practical and easy-to-implement intervention. In an environment of rising healthcare costs, simple and low cost methods to improve patient outcomes are needed. Using a visual reminder, such as was evaluated in this research, is also easily applicable patients and no special training, education, or investment is required.

\section{Conclusion}

This research cannot conclusively confirm the value of a visual reminder in changing patients' health behavior to manage their diabetes. Though the data is trending toward a beneficial role, the sample size was not sufficient enough to definitively draw such a conclusion. Nonetheless, when considering the clinically significant differences between the CG and VG with greater improvements in all measured variables, implementation of a visual reminder into diabetes management should be considered. This is certainly true when considering the reported influence and how simple and inexpensive it would be to do so.

\section{References}

1. Beckman JA, Creager MA, Libby P (2002) Diabetes and atherosclerosis: epidemiology, athophysiology, and management. JAMA 287: 2570-2581.

2. Saudek CD (2000) The challenge of diabetes management. The American Journal of Managed Care, vol. 6, pp. S664-S667.

3. Harris MI, Flegal KM, Cowie CC, Eberhardt MS, Goldstein DE, et al. (1998) Prevalence of diabetes, impaired fasting glucose, and impaired glucose tolerance in U.S. adults. Diabetes Care 21: 518-524.

4. ADA (2011) 2011 National Diabetes Fact Sheet.

5. CDC (2014) National Diabetes Statistics Report.

6. Zhang P, Zhang X, Brown J, Vistisen D, Sicree R, et al. (2010) Global healthcare expenditure on diabetes for 2010 and 2030. Diabetes Research and Clinical Practice 87: 293-301.

7. Wild S1, Roglic G, Green A, Sicree R, King H (2004) Global prevalence of diabetes: estimates for the year 2000 and projections for 2030. Diabetes Care 27: 47-53.

8. UKPDS (1998) Intensive blood-glucose control with sulphonylureas or insulin compared with conventional treatment and risk of complications in patients with type 2 diabetes (UKPDS 33). Lancet 352: 837-853.

9. Litaker D, Mion L, Planavsky L, Kippes C, Mehta N, et al. (2003) Physician - nurse practitioner teams in chronic disease management: the impact on costs, clinical effectiveness, and patients' perception of care. J Interprof Care 17: 223-237.

10. Surwit RS, van Tilburg MA, Zucker N, McCaskill CC, Parekh P, et al. (2002) Stress management improves long-term glycemic control in type 2 diabetes. Diabetes Care 25: 30-34.

11. Natarajan S, Clyburn EB, Brown RT (2002) Association of exercise stages of change with glycemic control in individuals with type 2 diabetes. Am J Health Promot 17: 72-75.

12. Rachmani R, Levi Z, Slavachevski I, Avin M, Ravid M (2002) Teaching patients to monitor their risk factors retards the progression of vascular complications in high-risk patients with Type 2 diabetes mellitus--a randomized prospective study. Diabetic Medicine 19: 385-392.

13. Rothman R, Malone R, Bryant B, Horlen C, Pignone M (2003) Pharmacist-led, primary care-based disease management improves 
Citation: Clobes TA (2017) Visual Reminders and Patient Compliance of Type 2 Diabetics. J Diabetes Metab 8: 736 . doi:

hemoglobin A1c in high-risk patients with diabetes. Am J Med Qual 18 51-58.

14. Rothman RL, Malone R, Bryant B, Shintani AK, Crigler B, et al. (2005) A randomized trial of a primary care-based disease management program to improve cardiovascular risk factors and glycated hemoglobin levels in patients with diabetes. Am J Med 118: 276-284.

15. Whitlock WL, Brown A, Moore K, Pavliscsak H, Dingbaum A, et al. (2000) Telemedicine improved diabetic management. Mil Med 165 579-584.

16. The Diabetes Care Management Program: Managing Diabetes for a Healthy and Active Life (2007).

17. Detweiler JB, Bedell BT, Salovey P, Pronin E, Rothman AJ (1999) Message framing and sunscreen use: gain-framed messages motivate beach-goers. Health Psychol 18: 189-196.

18. E. Kemps and M. Tiggemann (2007) Modality-specific imagery reduces cravings for food: an application of the elaborated intrusion theory of desire to food craving. J Exp Psychol: 13: 95-104.

19. Schneder TR, Salovey P, Pallonen U, Mundorf N, Smith NF, et al. (2001) Visual and auditory message framing effects on tobacco smoking. Journal of Applied Social Psychology, 31: 667-682.

20. Faul F, Erdfelder E, Lang AG, Buchner A, (2009) Statistical power analyses using $G^{\star}$ Power 3.1: tests for correlation and regression analyses. Behav Res Methods 41: 1149-1160.
21. Norman GR, Streiner DL (2000) Biostatistics: the bare essentials. (2nd edn), Hamilton, BC Decker, Ontario.

22. Hadjadj S, Duengler F, Barriere M, Mauco G, Coisne D, et al. (2005) Determination of $\mathrm{HbAlc}$ concentrations in patients with acute myocardial infarction: comparison of the DCA 2000 device with the HPLC method," Diabetes Metab 31: 290-294.

23. ADA (2016) Glycemic Targets. Diabetes Care 38: S39-S46.

24. Johnson HD, Sholcosky D, Gabello K, Ragni R, Ogonosky N (2003) Sex differences in public restroom handwashing behavior associated with visual behavior prompts. Perceptual \& Motor Skills 97: 805-810.

25. Fox TA, Berry B, Bielicki AK, DeLyser J, Pivonka E (2003) Increasing Fruit and Vegetable Intake Through Innovative Marketing. Nutrition Today 38: 21-24.

26. Inoue M, Yamamoto S, Kan H, Harada N (2005) Body mass index change after one year from a health examination and its relation to attitudes toward lifestyle change in Japanese male workers. J Occupational Health 47: 78-81.

27. Luszczynska A, Sobczyk A, Abraham C (2007) Planning to lose weight: randomized controlled trial of an implementation intention prompt to enhance weight reduction among overweight and obese women. Health Psychol 26: 507-512. 